\title{
Flexoelectricity in Low Densification Materials and its Implication
}

\author{
Longlong Shu ${ }^{1}$, Zehui Yong ${ }^{2}$, Xiaoning Jiang ${ }^{3}$, Zhengqiu Xie ${ }^{4}$, Wenbin Huang ${ }^{4}$ \\ ${ }^{1}$ School of Materials Science and Engineering, Qianhu Campus, Nanchang University, Nanchang \\ 330031, Jiangxi, China \\ ${ }^{2}$ Department of Applied Physics, The Hong Kong Polytechnic University, Hong Kong, China \\ ${ }^{3}$ Department of Mechanical and Aerospace Engineering, North Carolina State University, Raleigh, NC, \\ 27695, USA \\ ${ }^{4}$ The State Key Lab of Mechanical Transmissions, Chongqing University, Chongqing, 400044, China \\ Corresponding email: whuang@cqu.edu.cn
}

\begin{abstract}
Coupling between the strain gradient and the electric polarization, also named flexoelectricity, is a fundamental but often overlooked property in solid dielectrics. The past ten years has witnessed its great potential in sensing and actuating applications, especially in nanomaterials or nanostructures. The recently discovered giant flexoelectricity in barium strontium titanate (BST) perovskite above its Curie temperature has boosted the research interests on flexoelectricity to a new height. The possible interpretation of the enhanced flexoelectricity in BST was initially attributed to the non-crystalline polar-phases or polar nano-regions existing in the perovskites, then to the inner micro strain generated from the material densification process. In this paper, we analyzed the origin of flexoelectricity in this kind of materials by using both analytical mechanics method and finite element simulation method. Our results suggested the inner micro defect generated during the material densification process would weaken the flexoelectricity of the materials. The observed polarization in the studied materials was likely induced by other mechanisms rather than the pure flexoelectricity.
\end{abstract}

Keywords: (BaSr) $\mathrm{TiO}_{3}$, low densification material, flexoelectricity, sensing and actuating application. 


\section{Introduction}

Flexoelectric effect, referred to the transformation from mechanical strain gradient to electric polarization, has drawn numerous research interests due to its promising potential in sensing and actuating applications, especially at micro and nano scale [1-5]. This new electro-mechanical coupling effect is usually written in the form of [6]

$$
P_{i}=\mu_{i j k l} \frac{\partial S_{j k}}{\partial x_{l}}
$$

where $P_{i}$ is the induced polarization, $S_{j k}$ is the induced strain, $x_{l}$ is the dimension along $x$ axis, and $\mu_{i j k l}$ is the flexoelectric coefficients, a fourth rank tensor.

The concept of flexoelectricity was firstly originated from liquid crystals $[7,8]$. The irregularly shaped polarized molecules existing in liquid crystals can be re-orientated under the mechanical strain gradient stimulus and yield a distinctive distribution. Later, similar strain gradient induced polarization phenomena were also found in crystalline materials, especially in those perovskites $\mathrm{ABX}_{3}$ [9-12]. When the material is under an inhomogeneous strain, the B-site of the lattice would deviate from the original cubic center position and follow the strain gradient direction, thus generating a net dipole.

Compared with piezoelectric effect, one distinctive advantage of flexoelectric effect lies in the absence of centro-symmetry limitation $[13,14]$. In this case, conventional highly symmetrical materials where the piezoelectricity is rigorously forbidden, is possible to exhibit a net dipole moment. Nevertheless, it is noticed that theoretical values of flexoelectric coefficients were evaluated to be only in the order of $e / a\left(10^{-10} \mathrm{C} / \mathrm{m}\right)$, where $e$ is the electronic charge and $a$ is the crystal parameter $[15,16]$. This value is so small compared with the piezoelectric stress constants (with the typical value of 1-100 $\mathrm{C} / \mathrm{m}^{2}$ ), considering the case that the value of strain and strain gradient is comparable in quantity $[17,18]$. 
Therefore, at the early study stage, flexoelectricity in solid dielectrics was regarded to be trivial and even artificial.

However, as suggested by Tagantsev, enhanced flexoelectricity may exist in those high permittivity materials according to the formula $\mu=\gamma \varepsilon e / a$. where $\varepsilon$ is the dielectric permittivity and $\gamma$ is a constant with respect to the material property [19]. Later, it was found that in vicinity of the Curie temperature of several perovskites, the flexoelectric coefficients could approach as high as $10^{-6}-10^{-4}$ $\mathrm{C} / \mathrm{m}$, which is $4-5$ order of magnitude higher than the classical values [20-22]. On the other hand, thanks to the scaling effect of flexoelectricity, heterostructure thin films were reported to present significant flexoelectric polarization due to the giant strain gradient existing in the film thickness direction [23,24]. People gradually realized numerous mysterious phenomena that cannot be well interpreted at present time might be attributed to this strain gradient effect $[25,26]$. Now, flexoelectricity was considered as one of the fundamental properties in solid dielectrics. However, the critical issue regarding the mechanism of enhanced flexoelectricity has yet been fully understood. The possible interpretation of the enhanced flexoelectricity in perovskites was initially accounted for the non-crystalline polar-phases or polar nano-regions existing in the perovskites [27-30]. Recently, Damjanovic et al. contended that the origin of enhanced flexoelectricity was actually attributed to the inner micro strain generated from the material densification process, despite the on-going controversy [31,32]. This paper will study flexoelectricity in BST materials with various densifications, and hence explore the interplay of the material densification and the related flexoelectric performance.

\section{Methods and simulation procedures}

According to the classical elastic mechanics theory, for a material with nominal structure, the application of a uniform force will always result in a uniform strain. For a material with the trapezoid 
structure, the application of a uniform force will result in a strain gradient rather than a uniform strain. Typically, the strain gradient is always varied with the spatial coordinates. Hence, the average strain gradient must be employed to evaluate the flexoelectric performance. Suppose the strain $S$ is continuously varied with the axis $x$, for the specific position 1 and position 2 , the average strain gradient can be written as:

$$
\frac{\overline{\partial S}}{\partial x}=\frac{S_{1}-S_{2}}{x_{2}-x_{2}}
$$

Note that this expression is independent with the route between two positions. The average strain gradient can be zero even under a very large strain. Consequently, high flexoelectric polarization should be only related to large average strain gradient instead of large local strain gradient, when the flexoelectric coefficients are unvaried.

Consider a solid material with an internal mechanical stress distribution $q$ along the $x$ axis in the form of

$$
q=k_{1} x+k_{2}
$$

where $k_{2}$ denotes the constant part and $k_{1}$ stands for the macro stress gradient. Now, we suppose the investigated material contains some kind of defect which corresponds to a large local strain gradient. Without loss of generality, we only use pores to represent the defects so that the density of the material can be quantified by the number of pores. The simplified model is schematically shown in Fig. 1, where a pore with the radius of $a$ was considered. Based on the Saint-Venant principle, the contribution of geometry singularities for the far field status can be ignored. Therefore the stress distribution at the far field can be estimated by simply removing the pore, thus being the same as the intact materials without any defect. The stress tensors at point $A$ in the far field can be expressed as [33] 


$$
\left(\sigma_{x}\right)_{\rho=b}=0,\left(\sigma_{y}\right)_{\rho=b}=q,\left(\tau_{x y}\right)_{\rho=b}=0
$$

where $\sigma, \tau$ and $\rho$ are the normal stress, shear stress and polar radius, respectively. Adopting the coordinate transformation, the stress components in the polar coordinate can be written as

$$
\left(\sigma_{\rho}\right)_{\rho=b}=\frac{q}{2}-\frac{q}{2} \cos 2 \varphi,\left(\tau_{\rho \varphi}\right)_{\rho=b}=\frac{q}{2} \sin 2 \varphi
$$

where $\varphi$ is the polar angle. Substitute the value of $q$ by Eq. 3 we can get

$$
\begin{gathered}
\left(\sigma_{\rho}\right)_{\rho=b}=\frac{1}{2}\left(k_{1} b \cos \varphi+k_{2}\right)(1-\cos 2 \varphi)=\frac{1}{4}\left(2 k_{2}+k_{1} b \cos \varphi-k_{1} b \cos 3 \varphi-2 k_{2} \cos 2 \varphi\right) \\
\left(\tau_{\rho \varphi}\right)_{\rho=b}=\frac{1}{2}\left(k_{1} b \cos \varphi+k_{2}\right) \sin 2 \varphi=\frac{1}{4}\left(k_{1} b \sin \varphi+k_{1} b \sin 3 \varphi+2 k_{2} \sin 2 \varphi\right)
\end{gathered}
$$

The stress distribution can be solved by dividing the above boundary conditions into four parts according to the harmonic order of the polar angle:

$$
\begin{gathered}
\left(\sigma_{\rho 0}\right)_{\rho=b}=\frac{1}{2} k_{2},\left(\tau_{\rho \varphi 0}\right)_{\rho=b}=0 \\
\left(\sigma_{\rho 1}\right)_{\rho=b}=\frac{1}{4} k_{1} b \cos \varphi,\left(\tau_{\rho \varphi 1}\right)_{\rho=b}=\frac{1}{4} k_{1} b \sin \varphi \\
\left(\sigma_{\rho 2}\right)_{\rho=b}=-\frac{1}{2} k_{2} \cos 2 \varphi,\left(\tau_{\rho \varphi 2}\right)_{\rho=b}=\frac{1}{2} k_{2} \sin 2 \varphi \\
\left(\sigma_{\rho 3}\right)_{\rho=b}=-\frac{1}{4} k_{1} b \cos 3 \varphi,\left(\tau_{\rho \varphi 3}\right)_{\rho=b}=\frac{1}{4} k_{1} b \sin 3 \varphi
\end{gathered}
$$

where the number $(0,1,2$ and 3$)$ following the stress components denotes the multiplication of $\varphi$. Next we will obtain the solution for each part by using the stress potential method and applying the boundary conditions at the pore boundary. The solution of the first part can be directly written as:

$$
\sigma_{\rho 0}=\frac{k_{2}}{2} \frac{1-\frac{a^{2}}{\rho^{2}}}{1-\frac{a^{2}}{b^{2}}}, \sigma_{\varphi 0}=\frac{k_{2}}{2} \frac{1+\frac{a^{2}}{\rho^{2}}}{1-\frac{a^{2}}{b^{2}}}, \tau_{\rho \varphi 0}=0
$$

Since $b>>a$, we get $a / b=0$. The Eq. (12) can be further simplified as

$$
\sigma_{\rho 0}=\frac{k_{2}}{2}\left(1-\frac{a^{2}}{\rho^{2}}\right), \sigma_{\varphi 0}=\frac{k_{2}}{2}\left(1+\frac{a^{2}}{\rho^{2}}\right), \tau_{\rho \varphi 0}=0
$$

The second part can be solved by the semi-reverse method. Suppose $\sigma_{\rho 1}$ can be written as the 
product of $\cos \varphi$ with a function of $\rho$, while $\tau_{\rho \varphi 1}$ can be written as the product of $\sin \varphi$ with a function of $\rho$. We create an Airy stress potential function as $\Phi_{1}=f(\rho) \cos \varphi$ and get

$$
\sigma_{\rho 1}=\frac{1}{\rho} \frac{\partial \Phi_{1}}{\partial \rho}+\frac{1}{\rho^{2}} \frac{\partial^{2} \Phi_{1}}{\partial \varphi^{2}}, \sigma_{\varphi 1}=\frac{\partial^{2} \Phi_{1}}{\partial \rho^{2}}, \tau_{\rho \varphi 1}=-\frac{\partial}{\partial \rho}\left(\frac{1}{\rho} \frac{\partial \Phi_{1}}{\partial \varphi}\right)
$$

Considering the compatibility equation of elastic mechanics, we obtain

$$
\cos \varphi\left(\frac{\partial^{4} f(\rho)}{\partial \rho^{4}}+\frac{2}{\rho} \frac{\partial^{3} f(\rho)}{\partial \rho^{3}}-\frac{3}{\rho^{2}} \frac{\partial^{2} f(\rho)}{\partial \rho^{2}}+\frac{3}{\rho^{3}} \frac{\partial f(\rho)}{\partial \rho}-\frac{3 f(\rho)}{\rho^{4}}\right)=0
$$

The solution of $f(\rho)$ can be written as

$$
f(\rho)=C_{1} \rho+C_{2} \rho \ln \rho+\frac{C_{3}}{\rho}+C_{4} \rho^{3}
$$

where $C_{1}, C_{2}, C_{3}$ and $C_{4}$ are undetermined coefficients. Now the function $\Phi_{1}$ is in the form of

$$
\Phi_{1}=\left(C_{1} \rho+C_{2} \rho \ln \rho+\frac{C_{3}}{\rho}+C_{4} \rho^{3}\right) \cos \varphi
$$

The stress functions can be obtained as

$$
\begin{aligned}
& \sigma_{\rho 1}=\left(\frac{C_{2}}{\rho}-\frac{2 C_{3}}{\rho^{3}}+2 \rho C_{4}\right) \cos \varphi \\
& \tau_{\rho \varphi 1}=\left(\frac{C_{2}}{\rho}-\frac{2 C_{3}}{\rho^{3}}+2 \rho C_{4}\right) \sin \varphi
\end{aligned}
$$

Combining the boundary conditions

$$
\left(\sigma_{\rho 1}\right)_{\rho=b}=\frac{1}{4} k_{1} b \cos \varphi,\left(\tau_{\rho \varphi 1}\right)_{\rho=b}=\frac{1}{4} k_{1} b \sin \varphi,\left(\sigma_{\rho 1}\right)_{\rho=a}=0,\left(\tau_{\rho \varphi 1}\right)_{\rho=a}=0
$$

$C_{1}$ makes no contribution to the stress distribution. Only two independent equations can be obtained and three unknown parameters need to be solved. We can assume $C_{3}=0$, then the other two coefficients can be solved as $C_{2}=-\frac{a^{2} k_{1}}{4}, C_{4}=\frac{k_{1}}{8}$, the stresses can be derived as 


$$
\begin{gathered}
\sigma_{\rho 1}=\frac{k_{1} \rho}{4}\left(1-\frac{a^{2}}{\rho^{2}}\right) \cos \varphi \\
\sigma_{\varphi 1}=\frac{k_{1} \rho}{4}\left(3-\frac{a^{2}}{\rho^{2}}\right) \cos \varphi \\
\tau_{\rho \varphi 1}=\frac{k_{1} \rho}{4}\left(1-\frac{a^{2}}{\rho^{2}}\right) \sin \varphi
\end{gathered}
$$

The third part can be solved by the same method. The stress components can be derived as:

$$
\begin{aligned}
\sigma_{\rho 2} & =-\frac{k_{2}}{2}\left(1-\frac{a^{2}}{\rho^{2}}\right)\left(1-3 \frac{a^{2}}{\rho^{2}}\right) \cos 2 \varphi \\
\sigma_{\varphi 2} & =\frac{k_{2}}{2}\left(1+\frac{a^{4}}{\rho^{4}}\right) \cos 2 \varphi \\
\tau_{\rho \varphi 2} & =\frac{k_{2}}{2}\left(1-\frac{a^{2}}{\rho^{2}}\right)\left(1+3 \frac{a^{2}}{\rho^{2}}\right) \sin 2 \varphi
\end{aligned}
$$

The fourth part can be obtained as

$$
\begin{aligned}
& \sigma_{\rho 3}=-k_{1} \rho\left(\frac{a^{6}}{\rho^{6}}-\frac{5}{4} \frac{a^{4}}{\rho^{4}}+\frac{1}{4}\right) \cos 3 \varphi \\
& \sigma_{\varphi 3}=k_{1} \rho\left(\frac{a^{6}}{\rho^{6}}-\frac{1}{4} \frac{a^{4}}{\rho^{4}}+\frac{1}{4}\right) \cos 3 \varphi \\
& \tau_{\rho \varphi 3}=k_{1} \rho\left(\frac{a^{6}}{\rho^{6}}-\frac{1}{4} \frac{a^{4}}{\rho^{4}}+\frac{1}{4}\right) \sin 3 \varphi
\end{aligned}
$$

Therefore the complete expressions for the stresses distributions near the pore can be written as

$$
\begin{aligned}
& \sigma_{\rho}=\frac{k_{2}}{2}\left(1-\frac{a^{2}}{\rho^{2}}\right)+\frac{k_{1} \rho}{4}\left(1-\frac{a^{2}}{\rho^{2}}\right) \cos \varphi-\frac{k_{2}}{2}\left(1-\frac{a^{2}}{\rho^{2}}\right)\left(1-3 \frac{a^{2}}{\rho^{2}}\right) \cos 2 \varphi-k_{1} \rho\left(\frac{a^{6}}{\rho^{6}}-\frac{5}{4} \frac{a^{4}}{\rho^{4}}+\frac{1}{4}\right) \cos 3 \varphi \\
& \sigma_{\varphi}=\frac{k_{2}}{2}\left(1+\frac{a^{2}}{\rho^{2}}\right)+\frac{k_{1} \rho}{4}\left(3-\frac{a^{2}}{\rho^{2}}\right) \cos \varphi+\frac{k_{2}}{2}\left(1+\frac{a^{4}}{\rho^{4}}\right) \cos 2 \varphi+k_{1} \rho\left(\frac{a^{6}}{\rho^{6}}-\frac{5}{4} \frac{a^{4}}{\rho^{4}}+\frac{1}{4}\right) \cos 3 \varphi \\
& \tau_{\rho \varphi}=\frac{k_{1} \rho}{4}\left(1-\frac{a^{2}}{\rho^{2}}\right) \sin \varphi+\frac{k_{2}}{2}\left(1-\frac{a^{2}}{\rho^{2}}\right)\left(1+3 \frac{a^{2}}{\rho^{2}}\right) \sin 2 \varphi+k_{1} \rho\left(\frac{a^{6}}{\rho^{6}}-\frac{1}{4} \frac{a^{4}}{\rho^{4}}+\frac{1}{4}\right) \sin 3 \varphi
\end{aligned}
$$

The distribution of the normalized strain along the $x$ axis is plotted in Fig. 2. It can be easily observed that the strain distribution is symmetric to the $y$ axis while asymmetric to the $x$ axis. Large 
positive stress concentrations at two sides of the pore can be found, resulting into a nontrivial net strain gradient along the horizontal orientation, in other words, the macro stress gradient orientation. Accordingly, from the above analytical study, it can be predicted that strain gradient could redistribute near local defects inside the solid material which is under a macro strain gradient, leading to a possible change of the net strain gradient. The average macro effect induced by the randomly distributed local defects can be numerically estimated by the finite element method (FEM) introduced in the following part.

\section{Results and discussion}

In our FEM simulation, we built two conventional models as shown in Fig. 3, where the rectangle model acts as a reference. All the material properties of the two models are set to be identical. Moreover, we suppose the flexoelectric coefficients are constants and independent with the strain gradient. The material was selected as BST with its permittivity as high as 12000. It is noticed that all the models were set with the same boundary condition. For both the rectangle and trapezoid structures, the bottom side is mechanically clamped in the $z$ direction and the upper side was deformed with a $0.5 \%$ strain in the same direction. The movement along the transverse direction is allowed. The interplay of the material densification and the related flexoelectric performance (hereafter, the flexoelectric performance was actually characterized by the product of flexoelectric coefficients and average strain gradient) was simulated by including defects (pores). Furthermore, the material density is characterized by the size and number of the pores. The strain gradient response in the perfect rectangle and trapezoid structure was shown in Fig. 3a and 3d, respectively. It is obvious that only the trapezoid structure has an inhomogeneous strain distribution. Later we simulated the defect of the materials by including different pores. For the rectangle structure, large local strain gradient is concentrated on the vicinity of the pore. 
However, due to the geometry symmetry of the structure, the strain gradient will be cancelled along both $z$ and $x$ direction. As a result, no average strain gradient will be yielded. It is also worth mentioning that the flexoelectric performance is independent with the position and the number as well as the size of the pores. Hence, as shown in Fig. $4 \mathrm{~b}$ and 4c, the simulated average strain gradient is approaching zero.

Nevertheless, in the trapezoid structure, the result may be different. The strain gradually decreases from upper to bottom side of the structure. For a single pore, the strain is asymmetrical distributed around the vicinity of the pore. The strain gradient in the upper side of the pore is slightly larger than that at the bottom. In this case, the average strain gradient corresponds with the position of the pores. Here, we adopt a convergence simulation method to calculate the flexoelectric performance by eliminating the influence of the position of the pores. We firstly set the pores randomly distributed in the whole structure. The position of the pores was controlled by the random number generated by Matlab. In order to avoid the overlap of every two pores, we set the minimum distance between every two pores as 3 times of the radius of pores. Following the above criterion, we generate 1000 different patterns of random pores and apply FEM to calculate the average strain gradient of them. The final result shows a count distribution of 1000 strain gradient values, with an infinitesimal deviation. The statistic median was chosen for further analysis. In our simulation, we simulated the pore radius $R$ with the values of 6 $\mathrm{nm}, 8 \mathrm{~nm}, 10 \mathrm{~nm}$, and $12 \mathrm{~nm}$. On the other hands, the pore numbers was set to vary from 10 to 500 . Fig. $3 \mathrm{e}$ and Fig. $3 \mathrm{f}$ are only representatives of the trapezoid structure with pore number as high as 50 and 500 , respectively.

Surprisingly, as shown in Fig. 4, the statistic results suggested the average strain gradient will decrease when more pores are included in the structure. Moreover, this regulation is irrespective with the size of the pores. When the radius of pore were set to $12 \mathrm{~nm}$, the strain gradient slightly decreased 
with the numbers of the pores. However, when the radius of pore were set to $6 \mathrm{~nm}$, the strain gradient decreased even faster.

Next we used the number of pores to characterize the density of the materials and obtain the relationship between the flexoelectric property and the material density as shown in Fig. 5. Different with the previous understanding, giant flexoelectricity is more probable to exist in those material with high density rather than low densification materials. In such porous materials, even with the existence of large local strain gradient, the average strain gradient and related flexoelectricity was actually weakened. This result further proves that the artificial charge found in low densification materials were not attributed to flexoelectricity. ${ }^{27}$ Although lots of experimental and theoretical work done, the origin of flexoelectricity in BST material still retain much less understood. Recently, as suggested by one of our previous work, ${ }^{34}$ the thermal gradient could also induce the flexoelectric polarization and might be a possible explanation of the giant flexoelectricity in BST.

\section{Conclusions}

In conclusion, we systematically investigated the relationship between the flexoelectricity and local non-uniform strain gradient in BST materials, and further elucidated the possible relationship between the flexoelectricity and material densification (relative density). Based on the mechanic theory, we firstly analyzed the stress distribution in a non-uniform structure where a space pore is located at the center. The result suggested the stress will be inhomogenously distributed and lead to a net strain gradient due to its non-uniform structure. Subsequently, finite element method was employed in order to simulate the flexoelectricity in those porous BST materials, where different amounts of space pores were generated during the material densification process. Our results proved that the average strain gradient and related flexoelectricity in the BST materials consisting of more pores will be smaller than 
those consisting of few pores. Moreover, in view of the assumption in the paper, we further proved that low densification process would weaken the generated flexoelectricity. Our results would provide the theoretical guideline for the interpretation of the origin of enhanced flexoelectricity in this kind of materials.

\section{Acknowledgements}

L.S acknowledges extensive discussions with Prof. Yu Wang. This work is supported by the National Science Foundation of China under grant number 11604135 and 51605052, and in part by Science Foundation of Jiangxi Province (20161BAB216110). 


\section{References}

${ }^{[1]}$ U. Bhaskar, N. Banerjee, A. Abdollahi, Z. Wang, D. G. Schlom, A. J. H. M. Rijnders, G. Catalan, Nat. Nanotech. 11 (2016) 263-266.

${ }^{[2]}$ H. Lu, C. W. Bark, D. L. O. Esqu, J. Alcala, C. B. Eom, G. Catalan, A. Gruverman, Science 336 (2012) 59-61.

${ }^{[3]}$ L. Shu L, W. Huang, S. R. Kwon, Z. Wang, F. Li, X. Wei, X. Yao, S. Zhang, M. Lanagan, X. Jiang, Appl. Phys. Lett. 104 (2014) 232902.

${ }^{[4]}$ X. Jiang, W. Huang, S. Zhang, Nano Energy 2 (2013) 1079-1092.

${ }^{[5]}$ W. Huang, K. Kim, S. J. Zhang, F. G. Yuan, and X. N. Jiang, Phys. Status Solidi RRL 5 (2011) $350-352$.

${ }^{[6]}$ L. E. Cross, J. Mater. Sci. 41 (2006) 53-63.

${ }^{[7]}$ J. S. Patel, R. B. Meyer, Phys. Rev. Lett. 58 (1987) 1538-1540.

${ }^{[8]}$ P. Jacques P, P. S. Pershan, J. Appl. Phys 47 (1976) 2298-2312.

${ }^{[9]}$ P. Zubko, G. Catalan, and A. K. Tagantsev, Annu. Rev. Mat. Res. 43 (2013) 387-421.

${ }^{[10]}$ Y. Ling, X. Lu, J. Niu, H. Chen, Y. Ding, X. Qu, L. Zhao, J. Alloys Comp. 666 (2016) 23-29.

${ }^{[11]}$ S. Yu, J. Wei, S. Kang, J. Chen, G. Wu, J. Alloys Comp. 586 (2014) 328-332.

${ }^{[12]}$ H. Chen, W. Wang, Y. Li, P. Zhang, H. Nie, Q. Wu, J. Alloys Comp. 632 (2015) 23-29.

${ }^{[13]}$ L. Shu, X. Wei, T. Pang, X. Yao, and C. Wang, J. Appl. Phys. 110 (2011) 104106.

${ }^{[14]}$ P. V. Yudin and A. K. Tagantsev, Nanotechnology 24 (2013) 432001.

${ }^{[15]}$ A. K. Tagantsev, Phys. Rev. B 34 (1986) 5883-5889.

${ }^{[16]}$ P. Zubko, G. Catalan, A. Buckley, P. R. L. Welche, J. F. Scott, Phys. Rev. Lett. 99 (2007) 167601. 
${ }^{[17]}$ J. Tichy, J. Erhart, E. Kittinger, J. Privratska, Fundamentals of piezoelectric sensorics: mechanical, dielectric, and thermodynamical properties of piezoelectric materials, Springer (2010).

${ }^{[18]}$ A. K. Tagantsev, Sov. Phys. JETP 61 (1985) 2108-2122.

${ }^{[19]}$ W. Ma and L. E. Cross, Appl. Phys. Lett. 81 (2002) 3440-3442.

${ }^{[20]}$ L. Shu, X. Wei, L. Jin, Y. Li, H. Wang and X. Yao, Appl. Phys. Lett. 102 (2013) 152904.

${ }^{[21]}$ W. Ma and L. E. Cross, Appl. Phys. Lett. 88 (2006) 232902.

${ }^{[22]}$ G. Catalan, A. Lubk, A. H. G. Vlooswijk, E. Snoeck, C. Magen, A. Janssens, G. Rispens, G. Rijnders, D. H. A. Blank, and B. Noheda, Nat. Mater. 10 (2011) 963-967.

${ }^{[23]}$ B. C. Jeon, D. Lee, M. H. Lee, S. M. Yang, S. C. Chae, T. K. Song, D. B. Sang, S.C. Jin, G. Y. Jong and T. W. Noh, Adv. Mater. 25 (2013) 5643-5649.

${ }^{[24]}$ D. Lee, A. Yoon, S. Y. Jang, J. G. Yoon, J. S. Chuang, M. Kim, J. F. Scott, T. W. Noh, Phys. Rev. Lett. 107 (2011) 057602.

${ }^{[25]}$ Z. Wen, X. Qiu, C. Li, C. Zheng, X. Ge, A. Li and D.Wu, Appl. Phys. Lett. 104 (2014) 042907.

${ }^{[26]}$ A. I. Frenkel, Y. Feldman, V. Lyahovitskaya, E. Wachtel and I. Lubomirsky, Phys. Rev. B 71 (2005) 024116

${ }^{[27]}$ E. Wachtel and I. Lubomirsky, Adv. Mater. 22 (2010) 2485.

${ }^{[28]}$ S. Zeng, G. Ren, C. Xu, J. Alloys Comp. 509 (2011) 2540-2543.

${ }^{[29]}$ J. Narvaez and G. Catalan, Appl. Phys. Lett. 104 (2014) 162903.

${ }^{[30]}$ L. J. Dunne, M. Valant, A. K. Axelsson, G. Manos, N. M. Alford, J. Phys. D: Appl. Phys. 44 (2011) 375404.

${ }^{[31]}$ A. Biancoli, C. M. Fancher, J. L. Jones, D. Damjanovic, Nat. Mater. 14 (2015) 224-229.

${ }^{[32]}$ S. Hashemizadeh, A. Biancoli, D. Damjanovic, J. Appl. Phys. 119 (2016) 094105. 
${ }^{[33]}$ A. P. Boresi, R. J. Schmidt, Advanced Mechanics of Materials, John Wiley \& Sons (2012).

${ }^{[34]}$ T. Kim, W. Huang, S. Huang, X. Jiang, Appl. Phys. Lett. 108 (2016) 232902. 


\section{Figure captions:}

FIG. 1. Mechanic model of a solid material under a horizontal stress gradient with a space defect (pore) located at its center (denoted by the small circle with solid line), where $x, y$ is the coordinate, $a$ is the radius of the pore. A general point $A$ at the far field was employed to obtain the strain distribution near the pore area. Point $A$ is located at the distance of $b$ to the center of the pore with an angle of $\varphi$. The normal radial stress and shear stress components in the polar coordinate system at point $A$ are expressed as $\sigma_{\rho}$ and $\tau_{\rho \varphi}$, respectively. The assumed stress distribution at point $A$ is $q$ in the Cartesian coordinate system.

FIG. 2. Normalized strain distribution $\varepsilon_{x x}$ near a local pore within a solid material under a uniform macro mechanical stress gradient.

FIG. 3. (Color online) Strain gradient distribution between rectangle and trapezoid structure with different sizes and numbers of pores by FEM simulation. Fig. 4a, 4b, 4c stand for the rectangle structure with no pore, 50 pores, and 500 pores, respectively. Fig. 4d, 4e, 4f stand for the trapezoid structure with no pore, 50 pores, and 500 pores, respectively. Since the average strain gradient is related with the location of the pores, the pores generated by FEM is set randomly. The final average strain gradient is picked as the average value after 1000 iterations. Both the rectangle and trapezoid models are set with the same height. The value of the strain gradient is expressed by color bar.

FIG. 4. (Color online) Simulation results for the average strain gradient in trapezoid structure as a function of the number of the pores. The purple, yellow, blue and pink dots stand for the simulation when the pores injected in the trapezoid structure have a radius of $6 \mathrm{~nm}, 8 \mathrm{~nm}, 10 \mathrm{~nm}$ and $12 \mathrm{~nm}$, respectively.

FIG. 5. (Color online) Simulation results for the average strain gradient in trapezoid structure as a 
function of material density. The density of the material is quantified by the effective size of the structure. 


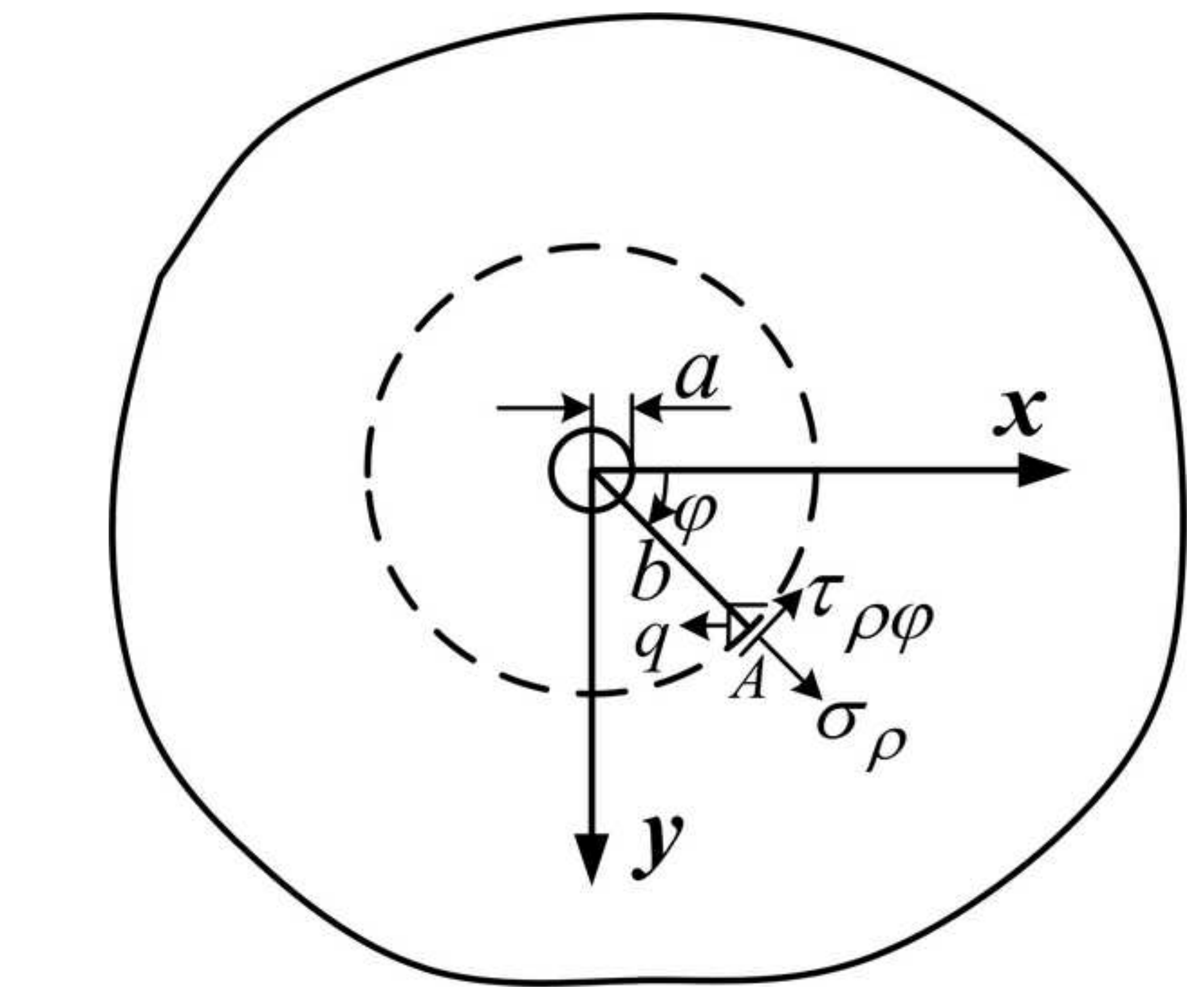



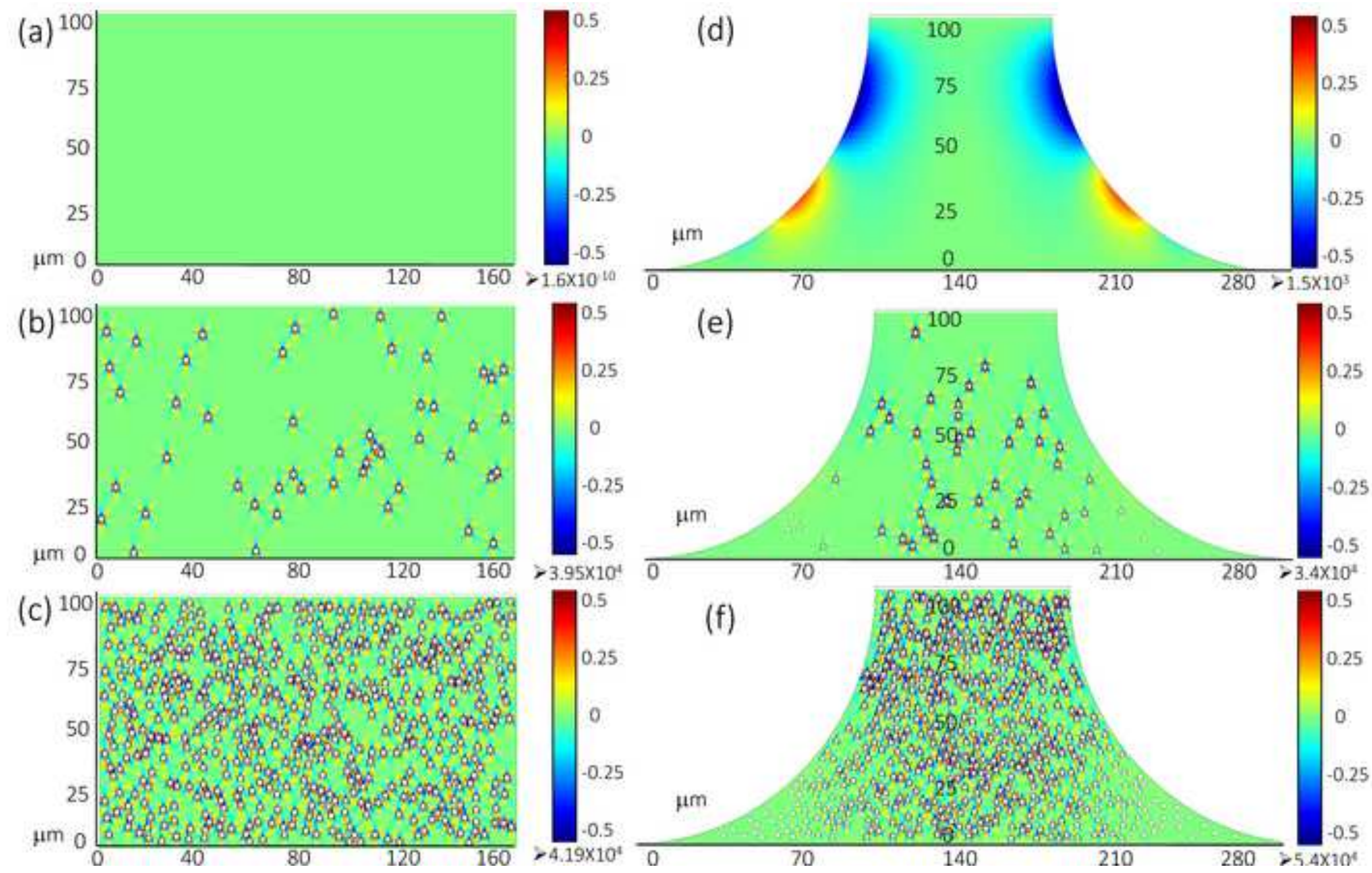


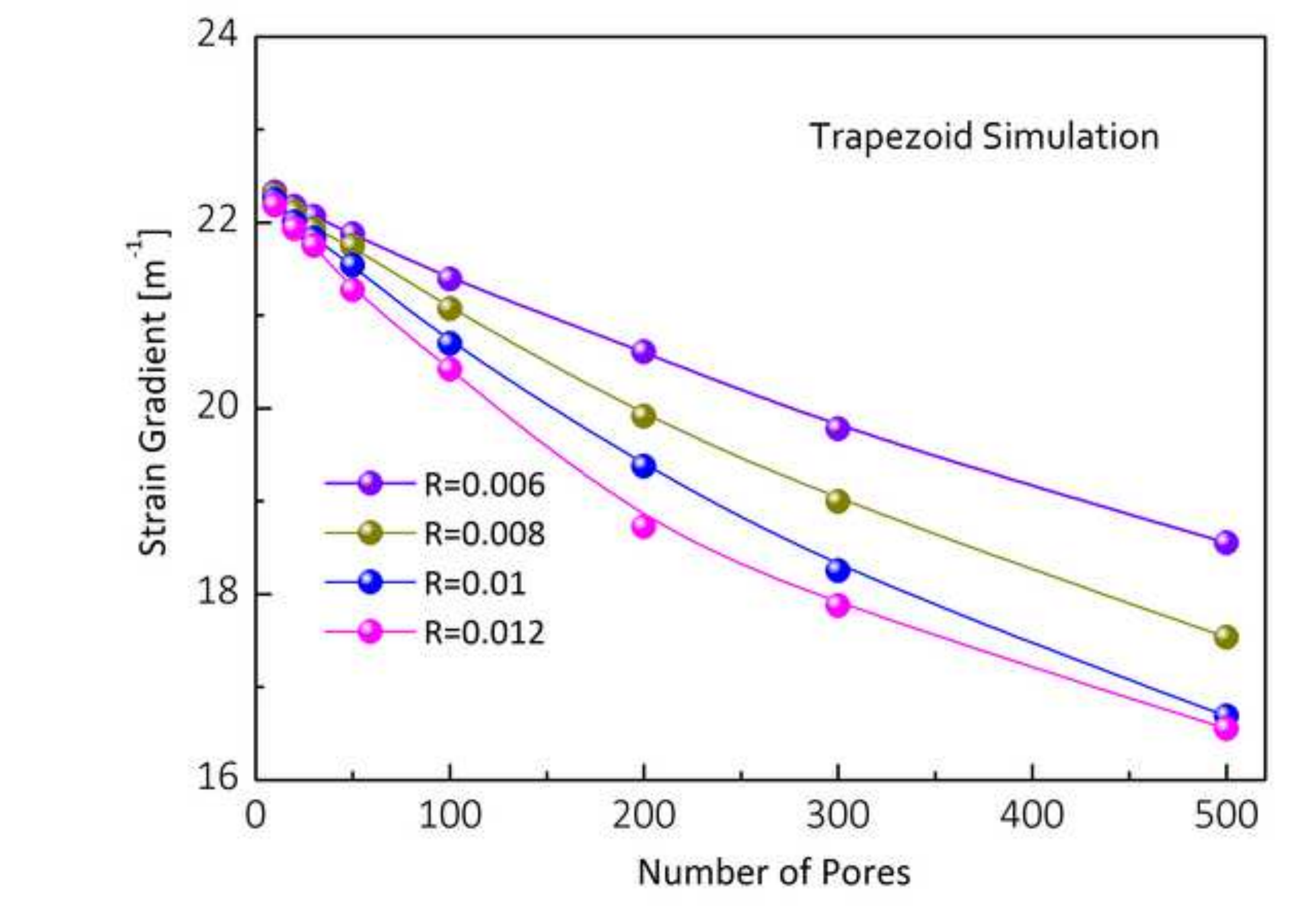




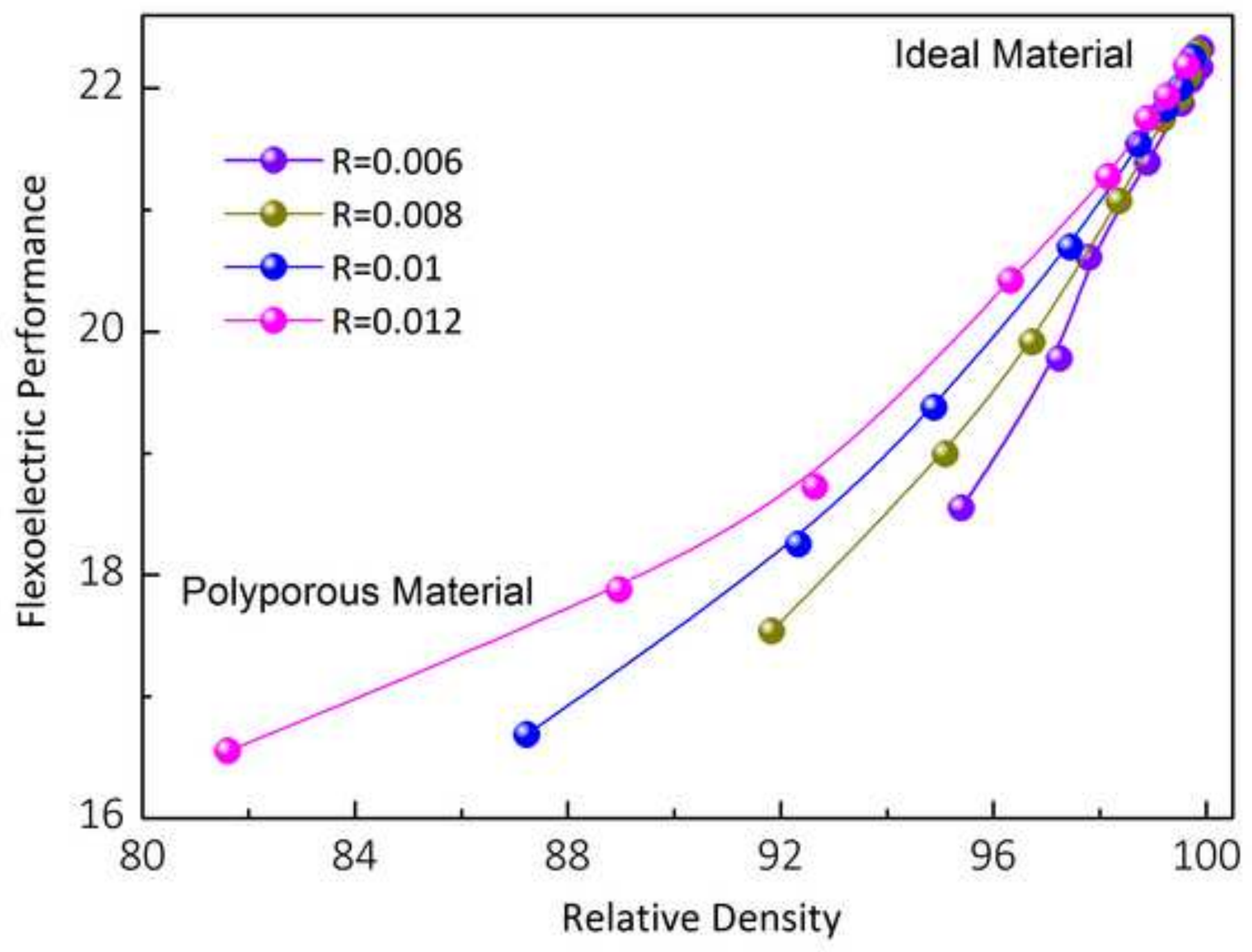

